\title{
Effects of Acute Exercise with Blood Flow Restriction on Oxidative Stress Biomarkers
}

\author{
Thiago Rozales Ramis ${ }^{1, *}$, Niara da Silva Medeiros ${ }^{1}$, Carlos Henrique de Lemos Muller ${ }^{2}$, \\ Francesco Boeno $^{2}$, Daiane Silveira ${ }^{1}$, Lisete Griebeler Souza ${ }^{1}$, Maristela Padilha de Souza ${ }^{1}$, \\ Claudia Funchal $^{1}$, Caroline Dani ${ }^{1}$, Jerri Luiz Ribeiro ${ }^{1}$ \\ ${ }^{1}$ Methodist University Center -IPA, Porto Alegre, RS, Brazil \\ ${ }^{2}$ Federal University of Rio Grande do Sul, Porto Alegre, RS, Brazil
}

\begin{abstract}
The aim of this study was to assess the effects of an acute session of resistance exercise with blood flow restriction in modulation of oxidative stress parameters at different moments. Eleven participants $(23.72 \pm 3.49$ years and $81.51 \pm 6.10 \mathrm{~kg}$ ) were recruited to perform a crossover design with three protocols: high intensity resistance exercise (HI $80 \%$ 1RM), low intensity resistance exercise (LI 30\% 1RM), and with blood flow restriction (LI-BFR 30\% 1RM). The oxidative profile in an acute session at four moments was assessed: baseline, immediately after exercise, and 24 and 48 hours later. The Ethics Committee at the Methodist University Center-IPA (Porto Alegre, Brazil) approved this study. The results did not show statistical significance ( $>0.05$ ) among moments and among groups for TBARS and protein carbonyls. Likewise, for the antioxidant activity there was also no difference $(\mathrm{p}>0.05)$ among moments and groups for sulfhydryl groups. The superoxide dismutase enzyme demonstrated a significant reduction $(\mathrm{p}<0.05)$ between baseline and immediately after exercise in the LI-BFR group. After group comparison, the HI group showed a significant increase $(p<0.05)$ in baseline and 24 hours when compared to the LI-BFR. An equal increase was observed in the HI group at the moment of 24 hours later when compared to the LI group. The catalase enzyme levels in the LI group were significantly higher than in the LI-BFR and HI groups at baseline and 24 hours $(\mathrm{p}<0.05)$ later. Lastly, the low intensity resistance exercise with blood flow restriction in maximum repetition appears not to have modified the oxidative profile in young and healthy subjects, suggesting that the hypertrophy mechanism of that methodology of training occurred in other molecular ways.
\end{abstract}

Keywords Reactive oxygen species, Resistance Training, Blood flow restriction

\section{Introduction}

Resistance training is an important method for prevention and maintenance of quality of life. Therefore, exercises with intensities between $60 \%-80 \%$ of one maximum repetition (1RM)(Walter R. \& Thompson, 2009) [1] are recommended for strength and hypertrophy improvements. Nevertheless, resistance training cannot be applicable for subjects who are unable to tolerate the mechanical stress generated in the joints. Thus, resistance training with blood flow restriction, also called "Kaatsu Training", arises as a possible training alternative (Loenneke et al., 2011; Sato, 2005) [2,3].

Evidence has demonstrated that resistance training with blood flow restriction provides strength and hypertrophy improvements even when performed with lower intensities (20\%-50\% 1RM) (Kubo et al., 2006; Loenneke et al., 2012; Patterson \& Ferguson, 2010) [4-6]. Moreover, it is clear in

* Corresponding author:

thiago.ramis@yahoo.com.br (Thiago Rozales Ramis)

Published online at http://journal.sapub.org/sports

Copyright (C) 2017 Scientific \& Academic Publishing. All Rights Reserved the literature that ischemia and reperfusion promote increase of reactive oxygen species related to a rise in xanthine oxidase activity during vascular hypoxia (Korthuis et al., 1985) [7].

Nevertheless, in spite of the increase in the number of articles demonstrating strength and muscle hypertrophy improvements in subjects that carry out low intensity resistance training with blood flow restriction, there are few articles reporting the effects of this method on oxidative stress biomarkers (Takarada et al., 2000; Goldfarb et al., 2008) $[8,9]$. On the other hand, it is well described that an acute session of resistance exercise with intensities near the maximum causes a rise in the blood markers of oxidative stress, furthermore, it can generate important adaptations in the antioxidant system (Powers \& Jackson, 2008; Finaud et. al., 2006; Bloomer \& Goldfarb, 2004; Bloomer et al., 2007) [10-13]. However, these acute and chronic adaptations are not found in all studies (Selamoglu et al., 2000) [14].

Therefore, the aim of this study is to assess the effects of an acute session of resistance exercise with blood flow restriction in the modulation of oxidative stress parameters at different moments. 


\section{Material and Methods}

Eleven physically active males were recruited for the study. The recruitment was done in the Methodist University Center-IPA. The participants had to answer the Physical Activity Readiness Questionnaire (PAR-Q). Participants of ages between 18 and 30 years, with a body mass index between $19-25 \mathrm{Kg} / \mathrm{m}^{2}$, who were classified as physically active through the IPAQ (Craig et al., 2003) [15] and who had not been involved in any resistance training in the previous six months were included. Exclusion criteria included the use of ergogenic resources, use of anabolic agents or anti-inflammatory drugs, history of cardiovascular, immune or inflammatory disease. The participants' characteristics can be observed in Table 1. This study is in accordance with the Declarations of Helsinki (1964) British Medical Journal (1964) ii, 177-178; and the Ethics Committee of the Methodist University Center-IPA, under the protocol number 364.202, approved this study. All participants were informed of the experimental procedures and possible risks before they gave written consent.

Table 1. Subject characteristics $(n=11)$ mean \pm SD

\begin{tabular}{cc}
\hline Variable & Subjects \\
\hline Age (years) & $23,72 \pm 3,49$ \\
Height $(\mathrm{m})$ & $1,75 \pm 0,02$ \\
Bíceps $(1 \mathrm{RM})$ & $18 \pm 3,19$ \\
Leg Press $(1 \mathrm{RM})$ & $172,52 \pm 29,20$ \\
Body mass $(\mathrm{kg})$ & $81,55 \pm 6,10$ \\
Fat mass $(\mathrm{Kg})$ & $20,87 \pm 3,84$ \\
Muscle mass $(\mathrm{Kg})$ & $37,51 \pm 3,63$ \\
\hline
\end{tabular}

$\mathrm{SD}=$ standard deviation, $1 \mathrm{RM}=$ one repetition maximum

During the first visit to the laboratory, the subjects answered the PAR-Q, and besides that the determination of blood flow restriction (BFR) and anthropometrics measurements were made. In the following visits, three familiarization sessions, separated by at least $48 \mathrm{~h}$, were done before the one repetition maximum test (1RM). After the $1 \mathrm{RM}$ test, volunteers came to the laboratory on three occasions, separated by at least one week, where they performed one of the three protocols: resistance exercise with high intensity (HI), low intensity resistance exercise with blood flow restriction (LI-BFR), and low intensity without blood flow restriction (LI). The protocols were made with $80 \% 1 \mathrm{RM}$ and $30 \% 1 \mathrm{RM}$, respectively. The order in which the protocols were to be carried out was illustration in the website randomization.com. Because of the exclusion of some volunteers, the sessions do not have the same number of participants. All the three protocols were performed between 8:00 and 10:00 A.M.. Before, immediately after, and 24 hours and 48 hours after exercise the blood samples were collected. This study employed a randomized crossover design.

The anthropometric assessment was made for subjects' descriptions using a plicometer (Mitutoyo with precision of $0,1 \mathrm{~mm}$ - Cescorf-Porto Alegre), a scale with coupled estadiometer (brand Welmy, with capacity for $200 \mathrm{~kg}$ and precision of $0,1 \mathrm{~kg}$ and $0,005 \mathrm{~m}$ ). The breadth measurements were made with a pachymeter and an anthropometer (Cescorf- Porto Alegre Brazil). The body composition was calculated using the five components methodology (Marfell-jones; Stewart, et. al. 2006) [16]. The evaluation techniques followed the standards of The International Society for the Advancement of Kinanthropometry (ISAK).

To determine the blood flow restriction, the volunteers attended the Laboratory of Exercise Physiology of the Methodist University Center-IPA in the morning. The participants remained in supine position during 20 minutes for determination of the arterial pressure at rest. The blood flow restriction used in the exercise was a blood pressure cuff (width $=17 \mathrm{~cm}$ and length $=70 \mathrm{~cm}$ ) for lower limbs, and (width $=15 \mathrm{~cm}$ and length $=50$ ) for upper limbs.

The maximum dynamic strength test was assessed through one maximum repetition test (1RM) of elbow flexion and leg press (table one). The volunteers performed a warm-up with dynamic movements followed by one series of 10RM in approximately $50 \% 1 \mathrm{RM}$. After three minutes, tests with a progressive load for $1 \mathrm{RM}$ were performed in five trials, using five minutes of rest between trials. The 1RM test consisted of the heaviest load that the participants could perform in one full range motion (concentric-eccentric phase) in one repetition.

The volunteers completed a food record of 24 hours (Fisberg et. al., 2009) [17] before the interventions and were advised to follow the same diet during every day of study participation so not to influence in the oxidative stress parameters being analyzed (Table 2). This food record was calculated to guarantee the supply of proteins, carbohydrates and fats consumed by subjects.

Table 2. Food record of the subjects $(\mathrm{n}=11)$ mean $\pm \mathrm{SD}$

\begin{tabular}{cc}
\hline Variable & Subjects \\
\hline Protein $(\mathrm{g})$ & $117,16 \pm 38,29$ \\
Carbohydrates $(\mathrm{g})$ & $302,56 \pm 80,07$ \\
Fat $(\mathrm{g})$ & $74,63 \pm 33,32$ \\
Kcal daily & $2350,55 \pm 690,37$ \\
\hline
\end{tabular}

$\mathrm{SD}=$ Standard deviation

The sessions of resistance exercise were conducted with elbow flexion and leg press exercises. The warm-up consisted of two sets of 15 repetitions using 50\% 1RM for each exercise. The HI group completed four sets of maximum repetitions using $80 \% 1 \mathrm{RM}$ without blood flow restriction with two minutes of rest between all sets. The LI-BFR group completed four sets of maximum repetition of each exercise using 30\% 1RM and blood flow restriction, as described above, with two minutes of rest between all sets. The LI group completed the same exercises, but without occlusion. A metronome controlled the duration of each repetition so that the participants completed the repetition in four seconds (two s. for the concentric and two s. for the eccentric phase).

In the biceps curl exercise, the cuff pressure was attached 
to the most proximal portion of the arm and inflated to $20 \mathrm{mmHg}$ below the systolic blood pressure. In the leg press exercise, occlusion occurred three minutes after the removing the cuff from the upper limbs, and then occlusion was performed at $20 \mathrm{mmHg}$ above the systolic blood pressure. These values are in agreement with the study by Goldfarb et. al. (2008) [9]. An oximeter (Nellcor NPB 195, Mallinckrodt Inc., St. Louis, USA) was used after each set to ensure that the blood flow was not completely halted. If the oximeter did not detect a pulse, the cuff pressure was reduced to five-ten $\mathrm{mmHg}$.

The blood samples were obtained at four moments: baseline, immediately after exercise, and 24 hours and 48 hours after exercise. Eight $\mathrm{ml}$ of venous blood without anticoagulant were collected. The samples were centrifuged at $1000 \mathrm{G}$ to obtain serum. The serum was aliquoted and frozen at $-20^{\circ} \mathrm{C}$ for further analysis.

The lipid peroxide was measured through the Reactive Substances to Thiobarbituric Acid method described by Wills [18], and the results were expressed in $\mathrm{nmol} / \mathrm{mg}$ protein. To determine the protein oxidation level, the technique used was that of measuring the level of oxidatively modified proteins: Protein Carbonyls, described by Reznick and Parker [19], and the results were expressed in $\mathrm{nmol} / \mathrm{mg}$ protein. The total sulfhydryl groups were measured through the technique described by Aksenov and Markesbery [19], and the results were expressed in $\mu \mathrm{M} / \mathrm{mg}$ protein. The antioxidant catalase enzyme (CAT) (Aebi, 1984) [21] and superoxide dismutase (SOD) (Misra \& Fridovich, 1972) [22] activities were also measured and expressed in UCAT/mg protein and USOD/mg protein, respectively.
The normal distribution was verified by Shapiro-Wilk test. The repeated measures analysis of variance (ANOVA) with Bonferroni post test was used. The level of significance was set at $p<0.05$. Analyses were conducted using the Statistical Package for Social Sciences (SPSS) 17.0.

\section{Results}

The descriptive characteristics of the participants and the food records with mean and standard deviation are shown in tables one and two, respectively.

Table 3 shows the baseline, post session, 24 and 48 hours results of the acute exercise on oxidative profile in low intensity with blood flow restriction (LI-BFR), high intensity (HI) and low intensity without blood flow restriction (LI) groups. There was no significant difference among moments and groups to lipid peroxide and protein carbonyls ( $>0.05$ ). In the antioxidant activity there was no significant difference among moments and groups for profile plasma concentrations of sulfhydryl groups. The SOD levels in LI-BFR group presented a significant reduction between the moments of baseline and post $(\mathrm{p}<0.05)$. When the HI group was compared to LI-BFR, it showed a significant increase in SOD levels at the post and 24 hours moments; moreover the HI presented a significant increase when compared to LI in the 24 hours moment $(\mathrm{p}<0.05)$. The catalase levels in LI group showed a significant increase when compared to LI-BFR and HI groups in baseline and 24 hours moments $(\mathrm{p}<0.05)$.

Table 3. Absolute values (mean \pm SD) for moments baseline, post, $24 \mathrm{~h}$ and $48 \mathrm{~h}$ in acute exercise of TBARS (nmoles/mg protein), Carbonyls (nmol/mg protein), sulfhydryls ( $\mu \mathrm{M} / \mathrm{mg}$ ), SOD (USOD/mg) and CAT (UCAT/mg). Low intensity resistance exercise with blood flow restriction (LI-BFR $\mathrm{n}=11$ ), High Intensity $(\mathrm{HI} n=10)$ and Low intensity without blood flow restriction $(\mathrm{BI} n=8)$

\begin{tabular}{|c|c|c|c|c|}
\hline \multirow{2}{*}{ Parameters } & \multicolumn{4}{|c|}{ Acute Exercise } \\
\cline { 2 - 5 } & Baseline & Post & $24 \mathrm{~h}$ & $48 \mathrm{~h}$ \\
\hline TBARS LI-BFR (nmoles/mg de pt) & $7,32 \pm 1,03$ & $6,05 \pm 0,72$ & $5,26 \pm 1,04$ & $6,34 \pm 1,39$ \\
\hline TBARS HI (nmoles/mg de pt) & $6,4 \pm 1,07$ & $6,29 \pm 1,24$ & $6,02 \pm 1,18$ & $6,94 \pm 1,85$ \\
\hline TBARS LI (nmoles/mg de pt) & $6,77 \pm 0,93$ & $6,47 \pm 1,03$ & $4,85 \pm 0,48$ & $6,06 \pm 2,27$ \\
\hline Carbonyls LI-BFR (nmol/mg de pt) & $65,11 \pm 25,72$ & $54,33 \pm 14,77$ & $63,94 \pm 18,98$ & $81,28 \pm 29,72$ \\
\hline Carbonyls HI (nmol/mg de pt) & $52,97 \pm 22,75$ & $69,65 \pm 26,81$ & $50,87 \pm 21,24$ & $67,66 \pm 32,4$ \\
\hline Carbonyls LI (nmol/mg de pt) & $63,07 \pm 29,53$ & $55,84 \pm 28,00$ & $63,62 \pm 26,35$ & $56,67 \pm 30,04$ \\
\hline Sulfhydryl LI-BFR $(\mu \mathrm{M} / \mathrm{mg})$ & $4,09 \pm 0,83$ & $4,23 \pm 1,17$ & $4,52 \pm 1,56$ & $3,88 \pm 1,47$ \\
\hline Sulfhydryl HI $(\mu \mathrm{M} / \mathrm{mg})$ & $4,43 \pm 1,39$ & $4,60 \pm 0,61$ & $3,92 \pm 0,91$ & $4,14 \pm 1,4$ \\
\hline Sulfhydryl LI $(\mu \mathrm{M} / \mathrm{mg})$ & $4,53 \pm 1,36$ & $4,39 \pm 0,64$ & $4,51 \pm 2,24$ & $4,32 \pm 0,26$ \\
\hline SOD LI-BFR $(\mathrm{USOD} / \mathrm{mg})$ & $1,23 \pm 0,58 \dagger$ & $0,37 \pm 0,15+\S$ & $0,87 \pm 0,64 \S$ & $1,09 \pm 0,86$ \\
\hline SOD HI $(\mathrm{USOD} / \mathrm{mg})$ & $1,24 \pm 1,01$ & $1,25 \pm 0,83 \S$ & $1,89 \pm 1,02 \S+\dagger$ & $1,39 \pm 0,84$ \\
\hline SOD LI $(\mathrm{USOD} / \mathrm{mg})$ & $1,13 \pm 0,78$ & $0,68 \pm 0,35$ & $0,50 \pm 0,05+\dagger$ & $1,58 \pm 1,28$ \\
\hline CAT LI-BFR $(\mathrm{UCAT} / \mathrm{mg})$ & $3,53 \pm 1,88 \S$ & $3,99 \pm 2,02$ & $2,95 \pm 0,86 \S$ & $4,13 \pm 1,61$ \\
\hline CAT HI $(\mathrm{UCAT} / \mathrm{mg})$ & $3,38 \pm 2,12+\dagger$ & $4,33 \pm 3,34$ & $5,21 \pm 2,35+\dagger$ & $3,95 \pm 2,16$ \\
\hline CAT LI (UCAT/mg) & $13,00 \pm 5,79 \S \dagger+\ddagger$ & $8,39 \pm 6,70$ & $13,67 \pm 13,00 \S \dagger+$ & $4,95 \pm 1,57 \ddagger$ \\
\hline
\end{tabular}

Significant difference in acute exercise among moments: $\uparrow$ Baseline and Post; $₫$ Baseline and $48 \mathrm{~h}$; Significant difference in acute exercise between groups $\S$ and $\dagger \dagger ;(\mathrm{p}<0,05)$. 


\section{Discussion}

The present study showed that a resistance exercise session of low intensity with blood flow restriction compared to high intensity and low intensity without occlusion exercises did not demonstrate differences in most of the oxidative stress parameters analyzed. Nevertheless, the high intensity exercise without blood flow restriction (HI) showed higher SOD enzyme activity than the low intensity exercise with blood flow restriction (LI-BFR). Furthermore, the CAT enzyme activity in low intensity without blood flow restriction (LI) presented a higher level than the other protocols at two moments: baseline and 24 hours later. This study is one of the first that assesses and compares the oxidative profile at baseline, post session, and 24 and 48 hours after an acute session of exercise.

The first study that demonstrated the effects of LI-BFR on oxidative stress markers was the Takarada et. al. (2000) [8] study. In this study, the authors did not verify a significant difference for lipid peroxidation after the session with LI-BFR. This corroborates with our results. However, in the study of Takarada et. al. (2000) [8] the protocol of exercise and sample (athletes) studied were different from the present study, hence the comparison became difficult.

According to Loenneke et. al. (2011), oxidative stress appears not to increase with LI-BFR in intensities below $30 \% 1 \mathrm{RM}$, regardless of the exercise performed [2]. In accordance with this, the results presented in this study did not indicate changes in plasma concentrations of the sulfhydryl groups in response to acute exercise with BFR, which demonstrate maintenance of repair capacity as a response to oxidative stress. In addition, that response was not different from other groups indicating that LI-BFR showed the same repair capacity of the HI despite the lowest intensity.

Plasmatic concentration of protein carbonyls (PC) are indirect biomarkers of protein damage. The present study does not show any statistical difference among groups. Goldfarb et. al. (2008) compared the effects of LI-BFR $(30 \%$ $1 \mathrm{RM}), \mathrm{HI}(70 \% 1 \mathrm{RM})$ and occlusion without exercise (BFR) [9]. The authors verified that there was a significant increase in plasmatic concentration of protein carbonyls (PC) in $\mathrm{HI}$ and BFR groups in comparison with LI-BFR. A study of Garten et. al. (2015) [23] also demonstrated a significant increase in $\mathrm{PC}$ in moderate intensity (MI 70\% 1RM) in comparison with LI-BFR. Taking into account the data presented above, it could be possible to state that LI-BFR does not generate higher protein damage than the same exercise performed without occlusion, even with a high intensity. Because of the not increased PC, it is possible that the LI-BFR reduces oxidative damage [9].

However, in the present study the antioxidant enzyme SOD reduces after the LI-BFR protocol, but increases in the $\mathrm{HI}$ protocol. The possible mechanism that explains this was reported by Afolayan et. al. (2014) [24]. The authors demonstrated that SOD dissociates from iHSP70 increasing its mitochondrial concentrations as the ATP production in the mitochondria increases. On the other hand, when the ATP concentrations are decreased and ADP concentrations are increased the SOD does not dissociate reducing its mitochondrial concentrations (Afolayan et al., 2014) [24]. Moreover, the authors reported that methylation in the promoter region of DNA to SOD-2 diminishes its expression (Afolayan et al., 2014) [24]. The reduction of the SOD activity after exercise with blood flow restriction was an unexpected response and its mechanism needs to be elucidated. This fact probably occurs because the exercise with blood flow restriction causes hypoxia. There are mitochondrial oxygen concentrations reductions that could reduce ATP production and increase ADP concentrations, therefore contributing to reducing SOD activity combined with hypermethylation of DNA. However, that is only speculation and the mechanism needs to be studied.

The CAT enzyme reduces hydrogen peroxide to water, thus avoiding the hydroxyl radical that can be extremely harmful to tissues (Powers \& Jackson, 2008) [10]. In this study, there was no statistical difference among moments in the three groups, but differences among groups were identified. This can be explained, since the baseline values of LI group were significantly higher than the other groups. At the moment 24 hours after exercise, the difference could be explained because the catalase enzyme has a greater activity in oxidative muscle fibers than in Type IIb muscle fibers [10]. Loenneke and colleagues (2010) suggest that a potential mechanism for low intensity resistance training with blood flow restriction generates results that are similar to strength and hypertrophy by recruiting type IIb fibers, albeit in low intensities [25]. Lastly, the expression of the catalase enzyme in anaerobic exercise is controversial (Powers \& Jackson, 2008; Fisher-wellman \& Bloomer, 2009) [10, 26]. Therefore, this could explain the CAT values in HI and LI-BFR protocols.

In general, exercises with BFR do not seem to generate greater oxidative stress than traditional training. According to Garten et. al., (2015) [23] this appears to occur because the continued arterial flow may change circulating antioxidants, reducing the accumulation in ROS proceeding from vasculature and muscle. However, this mechanism is debatable because the flow is constantly altered during the contractions with or without occlusion. Moreover, Goldfarb et. al. (2008) suggested an explanation for the unchanged oxidative stress in resistance training with blood flow restriction [9]. According to the authors the muscle contractions during exercise with blood flow restriction will be able to overcome the resistance of venous return, and then the oxidative stress markers would be removed from circulation [9]. We do not fully agree with this statement because overcoming resistance to venous return could generate a greater turbulent of blood flow causing greater biochemical activity with greater oxidative stress response. The explanation for an oxidative stress less than or equal to the high intensity exercise without occlusion remains speculative, requiring studies that verify each mechanism in more detail. 


\section{Conclusions}

Lastly, low intensity resistance training with blood flow restriction with maximum repetitions appears not to modify the oxidative profile in young and healthy subjects, suggesting from this point of view that vascular occlusion training is as safe as traditional strength training.

\section{ACKNOWLEDGEMENTS}

This work was funded by the Higher Education Personnel Training Coordination (Capes) and the Foundation for the Rio Grande do Sul State Research (Fapergs). We would like to thank the IPA Methodist University Center - (Porto Alegre, Brazil).

\section{REFERENCES}

[1] Walter R. Thompson NFG, Linda S. Pescatello, Kelli Allen. ACSM's Guidelines for Exercise Testing and Prescription American College of Sports Medicine. Eighth ed; 2009.

[2] Loenneke JP, Wilson JM, Wilson GJ, Pujol TJ, Bemben MG. Potential safety issues with blood flow restriction training. Scand J Med Sci Sports. 2011; 21(4): 510-8.S. Zhang, C. Zhu, J. K. O. Sin, and P. K. T. Mok, "A novel ultrathin elevated channel low-temperature poly-Si TFT," IEEE Electron Device Lett., vol. 20, pp. 569-571, Nov. 1999.

[3] Sato Y. The history and future of Kaatsu Training. Int J Kaatsu Training Res. 2005; (1): 5.

[4] Kubo K, Komuro T, Ishiguro N, Tsunoda N, Sato Y, Ishii N, et al. Effects of low-load resistance training with vascular occlusion on the mechanical properties of muscle and tendon. J Appl Biomech. 2006; 22(2): 112-9.

[5] Loenneke JP, Fahs CA, Rossow LM, Abe T, Bemben MG. The anabolic benefits of venous blood flow restriction training may be induced by muscle cell swelling. Med Hypotheses. 2012; 78(1): 151-4.

[6] Patterson SD, Ferguson RA. Increase in calf post-occlusive blood flow and strength following short-term resistance exercise training with blood flow restriction in young women. Eur J Appl Physiol. 2010; 108(5): 1025-33.

[7] Korthuis RJ, Granger DN, Townsley MI, Taylor AE. The role of oxygen-derived free radicals in ischemia-induced increases in canine skeletal muscle vascular permeability. Circ Res. 1985; 57(4): 599-609.

[8] Takarada Y, Nakamura Y, Aruga S, Onda T, Miyazaki S, Ishii N. Rapid increase in plasma growth hormone after low-intensity resistance exercise with vascular occlusion. J Appl Physiol. 2000; 88(1): 61-5.

[9] Goldfarb AH, Garten RS, Chee PD, Cho C, Reeves GV, Hollander DB, et al. Resistance exercise effects on blood glutathione status and plasma protein carbonyls: influence of partial vascular occlusion. Eur J Appl Physiol. 2008; 104(5): 813-9.
[10] Powers SK, Jackson MJ. Exercise-induced oxidative stress: cellular mechanisms and impact on muscle force production. Physiol Rev. 2008; 88(4): 1243-76.

[11] Finaud J, Lac G, Filaire E. Oxidative stress : relationship with exercise and training. Sports Med. 2006; 36(4): 327-58.

[12] Bloomer RJ, Goldfarb AH. Anaerobic exercise and oxidative stress: a review. Can J Appl Physiol. 2004; 29(3): 245-63.

[13] Bloomer RJ, Fry AC, Falvo MJ, Moore CA. Protein carbonyls are acutely elevated following single set anaerobic exercise in resistance trained men. J Sci Med Sport. 2007; 10(6): 411-7.

[14] Selamoglu S, Turgay F, Kayatekin BM, Gonenc S, Yslegen C. Aerobic and anaerobic training effects on the antioxidant enzymes of the blood. Acta Physiol Hung. 2000; 87(3): 267-73.

[15] Craig CL, Marshall AL, Sjostrom M, Bauman AE, Booth ML, Ainsworth BE, et al. International physical activity questionnaire: 12-country reliability and validity. Med Sci Sports Exerc. 2003; 35(8): 1381-95.

[16] Marfell-jones, T. O. M.; Stewart, A.; Carter, L. International standards for anthropometric assessment. International Society for the Advancement of Kinanthropometry., 2006.

[17] Fisberg RM, Marchioni DML, Colucci ACA. Assessment of food consumption and nutrient intake in clinical practice. Arquivos Brasileiros de Endocrinologia \& Metabologia. 2009; 53: 617-24.

[18] Wills ED. Mechanisms of lipid peroxide formation in animal tissues. Biochem J. 1966; 99(3): 667-76.

[19] Reznick AZ, Packer L. Oxidative damage to proteins: spectrophotometric method for carbonyl assay. Methods Enzymol. 1994; 233: 357-63.

[20] Aksenov MY, Markesbery WR. Changes in thiol content and expression of glutathione redox system genes in the hippocampus and cerebellum in Alzheimer's disease. Neurosci Lett. 2001; 302(2-3): 141-5.

[21] Aebi H. Catalase in vitro. Methods Enzymol. 1984; 105: 121-6.

[22] Misra HP, Fridovich I. The role of superoxide anion in the autoxidation of epinephrine and a simple assay for superoxide dismutase. J Biol Chem. 1972; 247(10): 3170-5.

[23] Garten, R. S., Goldfarb, A., Crabb, B., \& Waller, J. (2015). The impact of partial vascular occlusion on oxidative stress markers during resistance exercise. International journal of sports medicine, 36(07), 542-549.

[24] Afolayan AJ, Teng RJ, Eis A, Rana U, Broniowska KA, Corbett JA, et al. Inducible HSP70 regulates superoxide dismutase-2 and mitochondrial oxidative stress in the endothelial cells from developing lungs. Am J Physiol Lung Cell Mol Physiol. 2014; 306(4): 27.

[25] Loenneke JP, Wilson GJ, Wilson JM. A mechanistic approach to blood flow occlusion. Int J Sports Med. 2010; 31(1): $1-4$.

[26] Fisher-Wellman K, Bloomer RJ. Acute exercise and oxidative stress: a 30 year history. Dyn Med. 2009; 8(1): 1476-5918. 